\title{
Understanding the influence of stress on sedentary workers' sitting behavior in screen-based interaction context
}

\author{
Yang Chen \\ sonnechen95@gmail.com \\ Division of Industrial Design, National University of \\ Singapore \\ Singapore
}

\begin{abstract}
Sedentary work has become a significant part of a workday context, and this situation becomes more salient due to the COVID-19 pandemic. Existing studies show that body posture can be a good indicator of emotional states. However, to our knowledge, there are no studies that investigated the relationship between the characteristics of whole-body regions while seated and affective information related to stress for sedentary workers in screen-based working scenarios. This paper conducted a preliminary within-subjects study with eight participants performing three types of screen-based tasks at different difficulty levels for simulating natural working conditions. We developed a rapid posture coding technique to analyze sedentary workers' real-time sitting behavior and deployed multiple methods for continuously detecting their stress conditions. The results indicated that stress conditions and task determinants play an essential role in the postural changes of different body regions while seated. Our preliminary findings provide design implications and recommendations for developing a more unobtrusive health-promoting system in the real-life working context.
\end{abstract}

\section{CCS CONCEPTS}

\section{- Human-centered computing $\rightarrow$ Empirical studies in $\mathrm{HCI}$.}

\section{KEYWORDS}

Sitting posture; Stress; Screen-based interaction; Working context

\section{ACM Reference Format:}

Yang Chen and Ching Chiuan Yen. 2021. Understanding the influence of stress on sedentary workers' sitting behavior in screen-based interaction context. In Adjunct Publication of the 23rd International Conference on MobileHuman-Computer Interaction (MobileHCI '21 Adjunct), September 27-October 1,2021, Toulouse \& Virtual, France. ACM, New York, NY, USA, 5 pages. https://doi.org/10.1145/3447527.3474854

\section{INTRODUCTION}

Stress is a significant problem in modern society and an inescapable part of our daily lives [11]. According to a survey in European Union [19], stress, preceded by musculoskeletal disorders, has become the

Permission to make digital or hard copies of all or part of this work for personal or classroom use is granted without fee provided that copies are not made or distributed for profit or commercial advantage and that copies bear this notice and the full citation on the first page. Copyrights for components of this work owned by others than ACM must be honored. Abstracting with credit is permitted. To copy otherwise, or republish, to post on servers or to redistribute to lists, requires prior specific permission and/or a fee. Request permissions from permissions@acm.org.

MobileHCI '21 Adjunct, September 27-October 1, 2021, Toulouse \& Virtual, France

(C) 2021 Association for Computing Machinery.

ACM ISBN 978-1-4503-8329-5/21/09 . \$ \$15.00

https://doi.org/10.1145/3447527.3474854

\author{
Ching Chiuan Yen \\ didyc@nus.edu.sg \\ Division of Industrial Design, Keio-NUS CUTE Center, \\ National University of Singapore \\ Singapore
}

second most frequent occupational health issue among working Europeans. Built on the embodiment theory [21], studies showed that body posture could be a good indicator of emotional states. However, most studies mainly focused on some particular body parts, such as upper body posture [16, 20, 23]. To our knowledge, there are no studies investigate the characteristics of whole-body regions while seated and sedentary workers' stress conditions in screen-based office working scenarios. Considering the strong association between tasks requirements and posture $[9,14]$, the role of how different computing-based tasks play on office workers' sitting behavior and how the stress states and tasks intertwined with sitting behavioral shifts are rather under-explored.

This study aims to fill the gap by empirically exploring the impact of stress on sedentary workers' sitting behavior during screenbased computing tasks. To be more specific, this study is guided by the following research question: if sitting posture and postural shifts can potentially indicate sedentary workers' stress levels during different screen-based tasks? We conducted an exploratory controlled experiment with eight subjects performing screen-based tasks to mimic a real-life office working situation to investigate the research question aforementioned.

We designed three typical computer-based tasks (i.e., reading, typing and online meeting) and followed previous research work using mental workload as a main stress-inducing factor [3]. The reason behind this is that sedentary workers are inescapable to confront a certain amount of cognitive load during working situations. Thus, rather than deploying relax-inducing tasks (doing nothing or watching the calming video [11]), we designed mild cognitive load tasks as the control condition. To induce the mental stress, different task-related stressors (e.g., task difficulty, time limitation and ambient noise) were deployed for simulating the realistic working environment. Both subjective self-report stress data and objective EDA signals were extracted for more valid stress detection and analysis. To depict a holistic picture of how people sit in the working environment,a rapid posture coding technique was designed to manually code and classify sedentary workers' real-time sitting behavior unobtrusively. The results indicated that increased stress could significantly increase participants' head movements while performing text transcription tasks and increase arm movements while performing reading comprehension tasks. However, while performing online interview tasks, participants in mild cognitive load tend to make more postural shifts (i.e., leg, arm) than stress conditions. Our preliminary results highlighted that sitting behavior could provide extra information as complementary entities in stress detection and management. In particular, the main contributions of this exploratory study are as follows: 1 . We conduct a 
within-subject pilot study to explore how different cognitive loads influence sedentary workers sitting behavior while performing different screen-based tasks. 2. We discuss the insights and design implications of sitting posture as complementary entities as behavioral data for real-life stress monitoring system design. 3. We extend the knowledge of embodiment theory as a behavioral coping mechanism for stress detection and modulation.

\section{RELATED WORK}

This section provides an overview of studies that indicate a twoway relationship between posture and emotion and summarize existing approaches for stress measurement to ground and guide our research design.

\subsection{The association between motor actions and emotion}

Embodiment theory that muscular states are related to emotions has been widely studied [27]. Studies demonstrate that body expression is as powerful as facial expressions in conveying emotions and can even be more significant in revealing deception than facial expressions $[2,6,10]$. Greene et al.[13] identified that holding an upright posture is linked to a positive affective state and high confidence; conversely, slouched posture is considered a feature of depression and sadness. What is more, Wallbott et al.[26] claimed that body posture changes provide a strong indication of the differences in affective states.

Furthermore, research supporting embodiment theory highlights that postural body changes can affect stress response [17, 20, 25]. That is, participants with an upright posture during a psychological stress task reported better mood and lower stress than those manipulated with slumped sitting postures. Note that most studies mentioned above have concentrated on the movements of the upper body. The characteristics of whole-body regions are under-explored. Especially in screen-based interaction where the computing tasks highly restrict people's sitting posture, the lower body might reveal a great deal of information related to engagement and mental states. Therefore, in this exploratory study, we focused on investigating the association between characteristics of whole-body regions and stress conditions in screen-based interaction.

\subsection{Stress monitoring and measurement}

Researchers in behavioral, psychophysiological have been widely investigated in monitoring and measuring stress at work. Self-report questionnaires are widely used to measure stress levels in humans, and it is considered to be a much less intrusive and reliable method. Although studies indicated that self-reported instruments [8] were commonly used for stress measurement, they are restricted in offering information about the current stress state and fail to detect the subtle changes of an early stage of chronic stress. Computer vision techniques and facial Electromyogram (EMG) have been developed into automatic facial expression recognition for stress detection $[18,24]$. However, this method is highly dependent on environmental conditions and expensive hardware. In addition, behavioral responses such as facial expression can be easily faked by respondents. An alternative method to measure stress is through the direct measurement of the physiological signals reflected by the changes in heart rate, blood pressure, skin temperature, or electrodermal activity (EDA) [1]. This method has been widely applied in HCI as an unobtrusive way of objectively detecting participants' real-time stress changes [22].

\section{PRELIMINARY STUDY}

This study aims to explore whether sitting behavior can be used as a piece of behavioral information to reflect sedentary workers' stress conditions while performing screen-based tasks. Thus, we designed a within-subject experiment where participants performed three computing tasks under different cognitive load conditions.

\subsection{Participant}

A total of eight participants, five males and three females (age $=27 \pm 3.5$ ; $\mathrm{BMI}=24.1 \pm 1.74$; average task-related sitting hour per day $=7.5 \pm$ 0.75 ), were recruited using a convenient sampling method. Participants were excluded if they have any physical immobility or had ever experienced chronicphysical or musculoskeletal related disorders (MSDs) that required treatment or rest from normal activities for more than two days.

\subsection{Instruments}

Visual analog scale (VAS) [15]was administered to all participants before, between two experiment conditions (stress/control), and after experiment conditions. The scale is a $100 \mathrm{~mm}$ bipolar line ranging from $0=$ not stressed to $100=$ extremely stressed across a continuum. Empatica E4, a wearable device ( https://www.empatica.com/enint/research/e4/) combines multiple sensors to monitor physiological signals such as electrodermal activity (EDA), skin temperature was allocated to the participants for direct and objective measurement of their physiological responses associated with stress.

Rapid posture coding technique. Researchers in the field of non-verbal behavior have not yet established a generalized definition of how to define and classify postures [7]. For this study, instead of capturing the nuanced information, i.e., degree of inclination of people's postural changes, we aimed to depict how people sit in their working environment with a less intrusive and easy implementation method. We define the sitting posture as a whole body, including head, arms, trunk, and legs. Meanwhile, postural shifts are defined as a motion or a position shift for any aforementioned part of the human body. The rapid posture coding technique, see Table 1was designed based on Branton et al. [5]. Each sitting posture per second was categorized and coded into four different body parts: head, trunk, arm, and legs. Therefore, each sitting posture was represented as a set of four figures. For instance, Posture 1111 represents (Head upright free from support, trunk upright against the backrest, arms both on the desk and legs crossed).

\subsection{Experiment task}

To create an experiment environment that is close to real-life working scenarios, we designed three typical tasks:reading comprehension, text transcription, and online interview, which are commonly performed in the computer-related context under two different conditions: stress and control (mild cognitive load). The designated tasks are described as follows: 
Table 1: Rapid posture codebook

\begin{tabular}{|c|c|c|}
\hline & Description & Code \\
\hline \multirow{5}{*}{ Head } & Free from support (upright) & 1 \\
\hline & Free from support (lean right/left) & 2 \\
\hline & Against headrest (upright) & 3 \\
\hline & Against headrest (lean right/ left) & 4 \\
\hline & Supported by hand(s) & 5 \\
\hline \multirow{5}{*}{ Trunk } & Free from backrest (upright) & 1 \\
\hline & Free from backrest (lean right/left) & 2 \\
\hline & Against backrest (upright) & 3 \\
\hline & Against backrestAgainst backrest (lean right/ left) & 4 \\
\hline & Lounging (slumped back) & 5 \\
\hline \multirow{2}{*}{ Arms } & Upon desk (both) & 1 \\
\hline & Others & 2 \\
\hline \multirow{3}{*}{ Legs } & Free on the floor & 1 \\
\hline & Crossed & 2 \\
\hline & Others & 3 \\
\hline
\end{tabular}

Reading Comprehension(RC): Participants were asked to complete five reading comprehension practices in five minutes. During the stress condition, participants were provided with GRE reading comprehension tests. They were required to finish the tasks as quickly and correctly as possible in a noisy environment. When participants answered, the feedback of "wrong" was displayed as a stressor. During the control condition, participants were instructed to complete primary school reading comprehension practices. They were told that there is no time limitation for this task, however they would be stopped in five minutes.

Text transcription(TT): Participants were asked to transcribe an article in five minutes. During the stress condition, participants were provided with a scientific article with long terminologies.The text contained different font types and sizes to decrease the readability. Participants were asked to type as quickly and accurately as possible with a time limit and background noise. A timer and progress bar was also shown on the interface as a stressor.During the control condition, participants were asked to transcribe a reading paragraph from a primary school reading material. They were instructed to type as normally as possible without a time limitation.

Online Interview(OI): For this task, participants were required to attend a five-minute zoom meeting with one researcher. During the stress condition, participants were asked to complete a mental arithmetic task that takes TSST as a reference. During this calculation task, participants were asked to count backward from 2023 in steps of 17 . They were instructed to calculate as quickly and correctly as possible with noisy background music. If they miscalculated the number, they were asked to start again from the beginning. During the control condition, participants were encouraged to share something in their recent daily life (i.e., movies, food) in five minutes.

\subsection{Procedure}

Before the experiment, participants were given a cover story and were guided to fill in the research-made questionnaire. Each participant followed the four-phase experiment procedure, which lasted around $40 \mathrm{~min}$. In order to eliminate possible effects created by sequence and order effects, experiment conditions and three computing tasks were counterbalanced. The whole experiment was videotaped using a camera mounted on the tripod on the right side of participants to record their natural sitting behaviors continuously.

\subsection{Data collection and analysis}

Sitting postural data were analyzed by two coders derived from a video recording of participants' natural behavior during all the trials of the experiments using a behavioral coding software, Behavioral Observation Research Interactive Software (BORIS)[12]. Agreement on the coded data (i.e., identified posture and number of postural changes) was blind-validated through standard reliability measurements to achieve an acceptable level (>80\% agreement). The normality of data was confirmed with Shapiro-Wilk and analyzed in the Statistical Package for Social Sciences (SPSS-26.0) program.

\section{RESULT}

\subsection{Effectiveness of stress-induced tasks}

We used physiological signals combined with stress-perceived scales to measure the participants' stress levels. Self-reported stress derived from VAS was significantly higher after stress-induced tasks $(M=68 \pm 22)$ than results after control tasks $(M=28 \pm 10), p=0.001$, and there is no difference between the two baselines $(\mathrm{p}=0.358)$. We analyze physiological stress following Taylor et al.'s [17] method by using the automatic peak identification algorithm to compare the number of peaks during two conditions, and the result showed that participants overall have more peaks during stressed conditions, which in line with subjective data indicated that designated tasks elicited the expected stress.

\subsection{The most observed sitting posture affected by stress while performing different tasks}

Considering that different individuals have their habitual sitting behavior. We analyzed the most observed posture using multiple baseline methods. Although we conducted a case-by-case observational study, the discrepancy between most observed posture and stress levels while performing different screen-based tasks was found. Take P1 as an instance, Posture 5311 (Head supported by hand(s), trunk upright against the backrest, arms both on the desk and legs free on the floor) was observed most with $21.4 \%$ during stress-induced reading comprehension tasks, whereas Posture 1313 (head upright free from the headrest, trunk upright against the backrest, arms both on the desk and legs randomly put) was most observed with $31.9 \%$ during control reading comprehension tasks. These results yelled that customized postural detection systems should be designed in the future to better map people's sitting behavior in a real-life context.

\subsection{The postural shifts of different body parts affected by stress while performing different tasks}

Although overall postural shifts among all 8 participants in stressed conditions ( $M=45 \pm 20.6)$ were slightly higher compared to control conditions $(M=42.9 \pm 12.3)$, there was no significant difference 
between the two conditions, $\mathrm{t}(7)=0.583, \mathrm{p}=0.578$. There was a significant increase in postural shifts during the control OI $(M=27.5 \pm$ 7.5) compared to the stress-induced condition $(M=20.1 \pm 9.6), t(7)=$ $-4.538, \mathrm{p}=0.003$. Nevertheless, more postural shifts were observed in stressed conditions $(M=13.8 \pm 4.7)$ than control conditions $(M=9.1$ $\pm 3.6)$ in $T T, t(7)=2.772, p=0.028$. There was no significant effect for different stress levels in postural shifts while performing reading comprehension tasks, even though the number of postural shifts in the stressed condition $(M=11.1 \pm 9.8)$ was higher than control condition $(\mathrm{M}=6.3 \pm 4.2)$.

In addition, the characteristics of different body regions were affected by stress while performing different tasks. For Head, Mauchly's test indicated that the assumption of sphericity had been violated $(\mathrm{p}=0.003)$; therefore, we conducted repeated-measure ANOVA with Greenhouse-Geisser correction. There was a significant interaction between tasks and stress conditions $[\mathrm{F}(1.210,8.468)=11.531$, $\mathrm{p}=0.07]$. Post Hoc analysis with Bonferroni correction showed that head movement was significant on stress-induced $R C(p<0.001)$ and TT $(\mathrm{p}=0.002)$. For Arm, there was a significant effect on tasks $[\mathrm{F}(2,14)=30.124, \mathrm{p}<0.001]$ and a significant interaction between tasks and stress conditions $[\mathrm{F}(2,14)=13.671, \mathrm{p}=0.002]$. Post Hoc analysis showed that arm movement was significant on stress-induced OI $(\mathrm{p}<0.001)$ and RC $(\mathrm{p}=0.007)$. For trunk, a significant effect on tasks $[\mathrm{F}(2,14)=58.705, \mathrm{p}<0.001]$ and interaction between tasks and stress conditions $[F(2,14)=65.315, \mathrm{p}<0.001]$ were found. Post Hoc analysis with Bonferroni correction showed that trunk movement was significant on control OI $(\mathrm{p}<0.001)$. For trunk, a significant effect on tasks $[\mathrm{F}(2,14)=8.523, \mathrm{p}=0.004]$ was reported and Post Hoc analysis indicated that legs movement was significant on control OI $(\mathrm{p}=0.26)$.

\section{DISCUSSION}

Our motivation is to extract characteristics of sitting behavior for interpreting sedentary workers' stress conditions during computing tasks. The result derived from the pilot study demonstrated that participants tend to have different postural behavior during different stress conditions. Different computing tasks greatly influenced the characteristics (i.e., the number of postural shifts) of different body parts. For instance, tasks with reading and typing showed more postural shifts (i.e., head movements) in stress conditions. It can be explained that visual demand of these tasks highly influences participants' head position, like leaning forward. However, participants in the online interview showed more postural shifts like fidgeting in less stressful conditions. These results provide the initial evidence to support our hypothesis that stress can, to some extent,influence people's sitting behavior in screen-based interaction scenarios.

\subsection{Design implication}

Postural detection can be a more unobtrusive way for stress detection in a real-life working context. Our exploratory study indicated that stress levels could influence people's sitting postural behavior while performing different computing tasks which align with [7]. Thus, we envision that people's sitting behavior can be used as an indicator for interpreting sedentary workers' affective information related to stress in their working context. Apart from the classical psychological stress assessing method, postural behavior has great potential to pave the way for designing a more unobtrusive and practical real-life stress detection for sedentary workers. Although more and more technologies such as ECG, EDA, computer vision algorithms have been implemented for recognizing stress, it is undeniable that multimodal techniques for stress detection, physiological signals as well as behavioral responses, and other modalities should be considered to develop a much less intrusive health-promoting system for computing worker in an in-situ working scenario. We highlighted that physiological or behavioral changes as stress is not always reflected the same way among all individuals. Thus, a personalized system that can be self-adaptive to each individual should also be considered in a future study.

Taking the characteristics of whole body parts and tasks demand into consideration for the stress detection system. When considering the sitting postural behaviors as non-verbal signals for stress detection, our preliminary results indicated the importance of tasks and body units for postural detection. Studies in postural detection for interpreting affective information predominantly concentrated on the upper body, gesture, and facial expression [4], characteristics of whole body parts are underexplored into postural detection. Existing literature points out that [11] legs in many ways, which can reflect our genuine emotion and intention in real life. Especially in screen-based interaction, whether the upper body, such as arms, is occupied with specific computing tasks, the lower body can reveal a great deal of affective information. However, few studies have investigated how sitting behavior is affected by task determinants. In this study, we took the first step to explore the role of how stress conditions play on sedentary workers' whole body postural shifts and how stress states and tasks are intertwined with sitting postural behavior in computing workers. Our empirical results indicated that postural behavior of different body parts is significantly influenced by task demand and stress levels. Thus, we emphasized that it is important to take task determinants into consideration when using postural behavior as non-verbal signals for stress detection in a real-life working context.

The potential of postural shifts as a behavioral mechanism for stress regulation Our study provided preliminary evidence that affective information could lead to postural shifts among computing workers' working context. The converse effects of postural behavior on emotion modulation have been widely studied. Nair et al. [20] stated that upright posture had been associated with increased arousal and mood. Rather than static posture, researchers also found that dynamic posture such as walking posture could also affect people's emotion, which is in line with the embodied theories of cognition that physical body states influence emotional regulation. Inspired by embodied cognition theories, we envisioned that the connection between stress level and sitting posture is bi-directional that stress stimuli influencing sitting posture and posture intervention could affect stress perception. Thus, we suggested that future research can investigate whether posture intervention can be used as a behavioral mechanism for stress regulation strategy.

\section{2 limitation}

This preliminary study is cross-sectional, and the sampling size is limited, which could potentially lead to some noise and artifacts in either stress or sitting behavior data. The workplace is complex 
and consists of variations in terms of environment, interpersonal relationships, cultural structure, etc. Therefore, future studies with longitude study design and more participants are required to investigate the generalization of research outcomes to sedentary workers with computing tasks. Our approach to capture sitting behavior was to use a manually rapid posture coding system to annotate the video-based behavioral observation data with BORIS. Although this method is a common technique and easy to conduct, it failed to capture the more precise data of nuanced postural shifts, providing more accurate information of subjects' sitting behavior during different stress conditions. Therefore, additional posture detection techniques, e.g., pressure maps, should be applied to complement data for sitting posture analysis.

\section{CONCLUSION}

This study explored the association between characteristics of sitting posture and stress conditions during screen-based working situations. In particular, we conducted a within-subject study to analyze participants' sitting behavior while performing different computerized tasks at different stress levels. The result indicated that increased stress could significantly increase participants' head and leg movements while performing text transcription tasks and increase arm movements while performing reading comprehension tasks. However, while performing online interview tasks, participants in mild cognitive load tend to make more postural shifts (i.e., head, trunk) than stress conditions. These results from the pilot study provided preliminary support for the hypothesis that postural behavior can provide additional information about stress levels in computer users' working contexts. In addition, the results from our study highlighted that tasks should also be considered when applying postural behavior to extract information in the stress detection field. To the best of our knowledge, this work is the first to explore how stress states and tasks are intertwined with sitting posture in computing workers' working scenarios. By exploring the role of how characteristic of whole body parts while seated and different tasks play on sedentary workers' stress levels, it presented a promising tool to detect office workers' real-life stress in a more obtrusive and easy-to-implemented way.

\section{ACKNOWLEDGMENTS}

The authors are grateful for the assistance of Michelle Ho and Shen in shaping this paper and all participants for joining in this study.
[8] Sheldon Cohen, Tom Kamarck, Robin Mermelstein, et al. 1994. Perceived stress scale. Measuring stress: A guide for health and social scientists 10, 2 (1994), 1-2.

[9] DCAM Commissaris and K Reijneveld. 2005. Posture and movements during seated office work: results of a field study. In 37th Annual Conference on Ergonomics as a tool in Future Development and Value Creation, 10-12 October 2005, Oslo, Norway. 58.

[10] Paul Ekman and Wallace V Friesen. 1974. Detecting deception from the body or face. Fournal of personality and Social Psychology 29, 3 (1974), 288.

[11] Don Samitha Elvitigala, Denys JC Matthies, and Suranga Nanayakkara. 2020. StressFoot: Uncovering the Potential of the Foot for Acute Stress Sensing in Sitting Posture. Sensors 20, 10 (2020), 2882.

[12] Olivier Friard and Marco Gamba. 2016. BORIS: a free, versatile open-source event-logging software for video/audio coding and live observations. Methods in ecology and evolution 7, 11 (2016), 1325-1330.

[13] Shalom Greene, Himanshu Thapliyal, and Allison Caban-Holt. 2016. A survey of affective computing for stress detection: Evaluating technologies in stress detection for better health. IEEE Consumer Electronics Magazine 5, 4 (2016), 44-56.

[14] Liesbeth Groenesteijn, Peter Vink, Michiel de Looze, and Frank Krause. 2009. Effects of differences in office chair controls, seat and backrest angle design in relation to tasks. Applied ergonomics 40, 3 (2009), 362-370.

[15] Edward C Huskisson. 1974. Measurement of pain. The lancet 304, 7889 (1974), 1127-1131.

[16] Jung Won Kwon, Sung Min Son, and Na Kyung Lee. 2015. Changes in upperextremity muscle activities due to head position in subjects with a forward head posture and rounded shoulders. Fournal of physical therapy science 27, 6 (2015), 1739-1742.

[17] Darren M Lipnicki and Don G Byrne. 2008. An effect of posture on anticipatory anxiety. International fournal of Neuroscience 118, 2 (2008), 227-237.

[18] Daniel McDuff, Amy Karlson, Ashish Kapoor, Asta Roseway, and Mary Czerwinski. 2012. AffectAura: an intelligent system for emotional memory. In Proceedings of the SIGCHI conference on human factors in computing systems. 849-858.

[19] Malgorzata Milczarek, E Schneider, and E González. 2009. European agency for safety and health at work. OSH in figures: stress at work-facts and figures. Luxembourg: European Communities (2009).

[20] Shwetha Nair, Mark Sagar, John Sollers III, Nathan Consedine, and Elizabeth Broadbent. 2015. Do slumped and upright postures affect stress responses? A randomized trial. Health Psychology 34, 6 (2015), 632.

[21] Paula M Niedenthal. 2007. Embodying emotion. science 316, 5827 (2007), 10021005.

[22] Nargess Nourbakhsh, Yang Wang, Fang Chen, and Rafael A Calvo. 2012. Using galvanic skin response for cognitive load measurement in arithmetic and reading tasks. In Proceedings of the 24th Australian Computer-Human Interaction Conference. 420-423.

[23] Eva Ranehill, Anna Dreber, Magnus Johannesson, Susanne Leiberg, Sunhae Sul, and Roberto A Weber. 2015. Assessing the robustness of power posing: No effect on hormones and risk tolerance in a large sample of men and women. Psychological science 26, 5 (2015), 653-656.

[24] Anton Van Boxtel. 2010. Facial EMG as a tool for inferring affective states. In Proceedings of measuring behavior, Vol. 7. Noldus Information Technology Wageningen, 104-108.

[25] Lotte Veenstra, Iris K Schneider, and Sander L Koole. 2017. Embodied mood regulation: the impact of body posture on mood recovery, negative thoughts, and mood-congruent recall. Cognition and Emotion 31, 7 (2017), 1361-1376.

[26] Harald G Wallbott. 1998. Bodily expression of emotion. European journal of social psychology 28, 6 (1998), 879-896.

[27] Piotr Winkielman, Paula Niedenthal, Joseph Wielgosz, Jiska Eelen, and Liam C Kavanagh. 2015. Embodiment of cognition and emotion. (2015).

\section{REFERENCES}

[1] Ane Alberdi, Asier Aztiria, and Adrian Basarab. 2016. Towards an automatic early stress recognition system for office environments based on multimodal measurements: A review. Fournal of biomedical informatics 59 (2016), 49-75.

[2] Michael Argyle. 2013. Bodily communication. Routledge.

[3] Bert Arnrich, Cornelia Setz, Roberto La Marca, Gerhard Tröster, and Ulrike Ehlert. 2009. What does your chair know about your stress level? IEEE Transactions on Information Technology in Biomedicine 14, 2 (2009), 207-214.

[4] Abdelwahab Bourai, Tadas Baltrušaitis, and Louis-Philippe Morency. 2017. Automatically predicting human knowledgeability through non-verbal cues. In Proceedings of the 19th ACM International Conference on Multimodal Interaction. 60-67.

[5] P Branton and G Grayson. 1967. An evaluation of train seats by observation of sitting behaviour. Ergonomics 10, 1 (1967), 35-51.

[6] Peter E Bull. 2016. Posture \& gesture. Vol. 16. Elsevier.

[7] Justine Cassell, Yukiko I Nakano, Timothy W Bickmore, Candace L Sidner, and Charles Rich. 2001. Non-verbal cues for discourse structure. In Proceedings of the 39th Annual Meeting of the Association for Computational Linguistics. 114-123. 